\title{
Optimized Sampling Rate for Voltammetry-Based Electrochemical Sensing in Wearable and IoT Applications
}

\author{
Simone Aiassa ${ }^{12 *}$, Sandro Carrara ${ }^{23^{* *}}$, and Danilo Demarchi ${ }^{13^{* * *}}$ \\ ${ }^{1}$ Department of Electronics and Telecommunications, Politecnico di Torino, Turin, 10129 Italy \\ 2 Integrated Systems Laboratory, École Polytechnique Fédérale de Lausanne, Lausanne, 1015 Switzerland \\ ${ }^{3}$ Integrated Circuits Laboratory, École Polytechnique Fédérale de Lausanne, Neuchâtel, 2002 Switzerland \\ * Student Member, IEEE \\ ** Fellow Member, IEEE \\ *** Senior Member, IEEE
}

\begin{abstract}
The recent advancements in electrochemical measurements are guiding the development of new platforms for in-situ point-of-care monitoring of human-metabolite, markers and drugs. Despite this, the application of VoltammetryBased Sensing (VBS) techniques is still limited in wearable, portable, or loT systems. In order to use VBS approaches to measure analytes in small and low-power electronic platforms for diagnostics, several improvements are required. For example, the definition of a method to achieve the right trade-off between sample rate and sensing performance is still missing. To develop a method to define the best sampling rate, we present here an extensive analysis of experimental data to prove that is feasible to detect drugs such as paracetamol by Staircase Cyclic Voltammetry (SCV) or Differential Pulse Voltammetry (DVP) direct detection methods, with low sampling frequency. Our results prove that the proposed method helps the development of systems capable of discriminating the minimum pharmacology concentration of the metabolite under analysis with a massive reduction of the sampling frequency.
\end{abstract}

Index Terms-Bio-sensor, Cyclic Voltammetry, Differential Pulse Voltammetry, Electrochemical Sensors, Low Power, loT, Paracetamol, Point-of-care, Sampling Rate, Wearable.

\section{INTRODUCTION}

The real-time point-of-need biochemical sensing provides the possibility of direct non-invasive monitoring of patients, giving continuously valuable insights into their health and their well-being. Electro-analytical measurements can be directly exploited in-situ and without sample purification. This brings the benefit of a point-of-care detection of several different analytes and drugs on wearable, portable, and IoT systems [1]. If we consider this new scenario, the portable devices strongly constraint the maximum power consumption of the system. In order to enlarge the battery life-time, one possible approach is the reduction of the sampling rate of the sensing node [2], leading to a reduction of data density and signal bandwidth. In fact, the key components of the power consumption in the device are related to the power spent by signal elaboration and data transmission, which are both directly influenced by the sampling rate.

Potentiometric and amperometric techniques, for example, OpenCircuit Potential (OCP) [3] and Chrono-Amperometry (CA) [4], are usually exploited in wearable devices for electrochemical sensing thanks to their capability of carrying out the bio-sensing information through a low-complexity electrical-converter. In those cases, the sampled information (concentration of analyte), is directly related to an electrical quantity, voltage or current. The ratio between the number of samples and the number of information detected is one, implying an information throughput equal to one. Nevertheless, OCP and CA are not suitable for the detection of all the possible analytes, due to the higher variability and the poor stability over time of micro

Corresponding author: S. Aiassa (e-mail: simone.aiassa@polito.it) reference electrodes [5].

The voltammetry-based electro-analytical tools are largely used in electrochemistry to obtain quantitative information on RedOx processes in a laboratory environment [6]. Thanks to the recent advancement in electronics, the Voltammetry Based Sensing (VBS) techniques are appearing on the scene as methods for direct sensing at the point-of-need [7]-[9]. In VBS, the electrochemical cell is excited by a voltage sweep applied between its Working Electrode (WE) and its Reference Electrode (RE). The current produced by the oxidation or the reduction of the target species is then measured from the Counter Electrode (CE) or equivalently from the WE. This current usually is reported versus the applied potential obtaining the so-called voltammogram. The voltammogram has a peculiar shape that depends, for example, on the drug present in the solution with current peaks corresponding to RedOx phenomena. From the height of these peaks, it is so possible to evaluate the concentration of the drug [10]. The indirect method of measuring through peakdetermination in VBS introduces complexity and increases the data bandwidth (w.r.t. OCP and CA). The throughput is no more one: to sense one sample of concentration, we have to sample more than one time the current. However, the higher complexity of the method increases specificity and sensitivity [10] and provides simultaneous determination of different compounds [11]. To be effective, the VBS approach requires the sampling of a well-defined voltammogram. Despite the massive literature, at the best of our knowledge, a real definition of the optimal sampling rate does not exist. This value is usually set by empirical methods, for example, considering how many data points are forming the complete voltammogram graph. Therefore, as already suggested by [12]-[14], there is the need for a novel and effective method for reducing and optimizing the sampling 
rate in VBS.

The preliminary results here presented are necessary to quantitatively prove that the data produced by VBS techniques can be efficiently down-sampled. The final results demonstrate the efficient application of a sampling rate optimization method on a full set of in-vitro measurements, considering as test case the sensing of acetaminophen, also known as paracetamol and $\mathrm{N}$-acetylp-aminophenol (APAP), a widely used analgesic [15].

\section{A. Staircase Cyclic Voltammetry and Differential Pulse Voltammetry}

[12]-[14] consider the Fast Scan Cyclic Voltammetry (FSCV) for neurotransmitters, a specific case where the sample rate is limited by communication bandwidth. Differently, to the best of our knowledge, for the first time, we here investigate more general methods like Staircase Cyclic Voltammetry (SCV) and Differential Pulse Voltammetry (DPV) for drug monitoring.

The APAP can be sensed either through SCV or DPV with simple devices for continuous monitoring 1 describes those methods. The SCV (Fig. 1a) procedure requires the application of a driving voltage ramp between WE and RE, that generally is generated through a staircase of voltage step, defined by a voltage height $\left(\mathrm{V}_{\text {step }}\right)$ and a time length $\left(\mathrm{t}_{\text {step }}\right)$. The voltage scan ranges in an interval related to the RedOx process under analysis. The ratio between $V_{\text {step }}$ and $t_{\text {step }}$ is also called Scan Rate (SR). Every sampling time interval $t_{\text {sample }}$, a current sample is acquired from the CE/WE loop. According to the Randles-Sevcik equation [10], is possible to derive the analyte concentration by processing the voltammogram to detect the height of the current peak [6]. The DPV (Fig. 1b) adds to the staircase a pulse wave signal and introduces as main advantages the reduction of non-Faradic current related to double layer effect, the enhancement of the current peak, and the reduction of noise [16]. Typically, SCV is applied to detect both oxidation and reduction peaks, while DPV only for one kind of reaction, for this reason, the voltage-scan ranges can be different in the two methods.

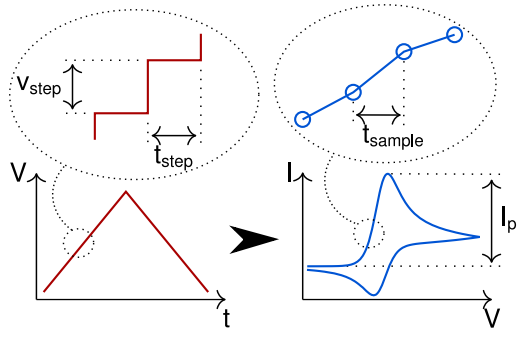

(a)

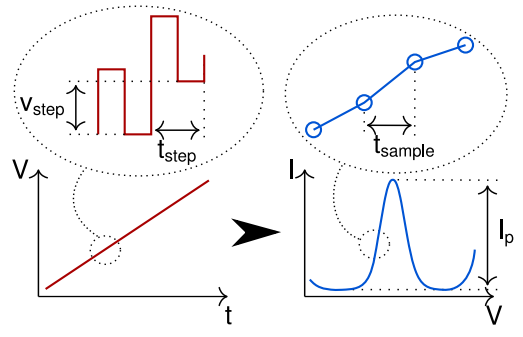

(b)

Fig. 1. SCV (a) and DPV (b) methods examples, the cell is driven by the wave (right) to acquire the voltammogram (left). The output can be elaborated in the calibration curve (c).
From electrochemical determination, is possible to derive a calibration curve (Fig. 1c) which is the relation between the concentration of analyte and the current (in VBS the height of the current-peak). Typically, the calibration curves form of a straight line in the region of interest, and the Sensitivity (S) represents the slope of this curve. The parameter Limit of Detection (LOD) specifies the minimum quantity of an analyte that can be determined.

\section{METHODS}

\section{A. Materials and Equipment}

APAP powder from Sigma Aldrich ${ }^{\circledR}$ (Acetaminophen BioXtra, $\geq 99 \%$ ) in the day of use is dissolved in a background electrolyte, namely, Phosphate Buffer Saline (PBS) solution at $\mathrm{pH}$ 7.4. The buffer is purchased from Sigma Aldrich ${ }^{\circledR}$ and it is composed of $10 \mathrm{mM}$ phosphate buffer, $2.7 \mathrm{mM}$ potassium chloride, and $137 \mathrm{mM}$ sodium chloride. The sensing equipment is composed of a commercial potentiostat, namely the Metrohm Autolab PGSTAT 302N, driven by the software Nova 1.11. The instrument is interfaced using DropSens DRP-110, Screen-Printed Electrode (SPE) composed of carbon WE with $4 \mathrm{~mm}$ diameter, carbon CE, and silver (pseudo-) RE [17] for the drug-detection.

\section{B. In Vitro Data Collection}

For the preliminary analysis, a sample containing $300 \mu \mathrm{M}$ of APAP is selected as a reference measure, since this value is the maximum in the therapeutic range [15]. A parametric analysis is performed in SCV considering a different number of samples per voltammogram, equivalently, varying the sampling frequency $\left(\mathrm{F}_{\mathrm{S}}\right)$. The SR is set to $0.2 \mathrm{~V} / \mathrm{s}$, the driving voltage ranges in $-0.1: 1.1 \mathrm{~V}$, while the step voltage height ranges in $1: 24 \mathrm{mV}$, and the step time length ranges in 5:120 ms. For the final analysis, a full sevenpoints calibration procedure in the therapeutic range $50: 300 \mu \mathrm{M}$ is performed. Subsequent increasing concentration steps of $50 \mu \mathrm{M}$ of APAP are measured. To consider the inter-electrode variability and to avoid artifacts, the measurement is repeated three times for each condition with a new electrode. The SCV is performed with a SR of $0.2 \mathrm{~V} / \mathrm{s}$, with a driving voltage ranging in $-0.1: 1.1 \mathrm{~V}$, with a step voltage height of $6 \mathrm{mV}$, and a step time length of $30 \mathrm{~ms}$. The DPV is performed at $0.1 \mathrm{~V} / \mathrm{s}$, in the range $0: 1 \mathrm{~V}$, with a step height of $6 \mathrm{mV}$, an step length of $60 \mathrm{~ms}$, the pulse amplitude is set to $60 \mathrm{mV}$, and the pulse time length to $30 \mathrm{~ms}$.

\section{Peak Detection and Elaboration}

The collected data are processed in Matlab $^{\circledR}$ (v. R2017b). After subtracting the background-current obtained by the blank measurement, the peaks are detected and analysed with the findpeaks built-in function to return the height of the oxidation-current peak and its position. The calibration curve, the sensitivity and the coefficient of determination $\left(\mathrm{r}^{2}\right)$ are derived by regression built-in function. The LOD is computed as three times the standard deviation of the blank measure around the peak, divided by $\mathrm{S}$. 


\section{RESULTS AND DISCUSSION}

\section{A. Preliminary Analysis}

Considering the main parameters of SCV and DPV methods as the Scan Rate (SR), the total number of samples acquired per ramp $\left(\mathrm{N}_{\mathrm{S}}\right)$, and the full voltage scan interval $\left(\mathrm{V}_{\text {scan }}\right)$, is possible to define the sampling frequency $\left(F_{S}\right)$ as in (1). The equation gives us three degrees of freedom to reduce the sampling rate, but, considering a fixed analyte and a fixed electrochemical sensor, only the reduction of the number of samples seems easy practicable. The voltage scan interval is determined by the reaction under analysis and also the $\mathrm{SR}$ is a variable which should be primarily optimized considering the diffusivity of the reaction under analysis [18].

$$
F_{S}=S R \cdot \frac{N_{S}}{V_{\text {scan }}}
$$

As a preliminary analysis, a set of lab measurements is considered varying the number of steps per SCV, fixing all the other parameters. Fig. 2 presents graphically the results. In Fig. 2 a the y-axis shows the peak-current error. The error is defined as the difference between the best possible estimation of the peak we can dispose of (i.e. sampling the current with the maximum sampling frequency granted by the instrument [19]) and a given approximation of the estimated peak (i.e. sampling the current with a given frequency, $x$-axis). The error quasi-linearly depends on the sampling period. This effect is due to the lower resolution in the peak measurement, but it is also highly influenced by the increase of voltage step height [20]. Fig. $2 b$ analyzes the Signal to Noise Ratio (SNR, y-axis) versus the sampling frequency. Two different values are taken into account: the SNR of the full voltammogram, and the SNR of only the detected output, i.e. the SCV peak value. The SNR is defined considering the signal $(\mathrm{S})$ as the full voltammogram or the detected peak at the maximum possible sampling rate, while the noise $(\mathrm{N})$ is the difference between the signal (S) and the output voltammogram or peak at the given sampling frequency. The noise introduced by the reduction of sampling frequency is visibly lower considering the SCV-peak with respect to the noise present in the full voltammogram. This happens because the peak detection is not a linear operator, but a feature-extractor, acting to some extent as a filter. The output in which we are interested to develop the electrochemical sensor is primarily the peak height. This analysis proves that is feasible to reduce of the sampling rate over what is usually considered the limit because the noise introduced in the voltammogram does not reflect linearly to the increase of noise in the output measure.

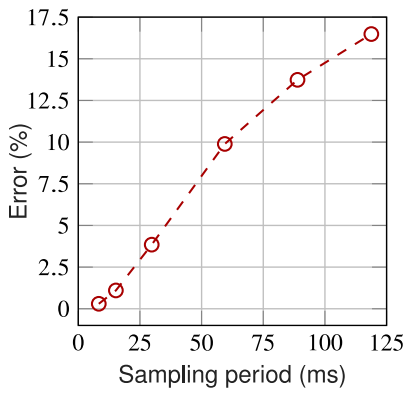

(a)

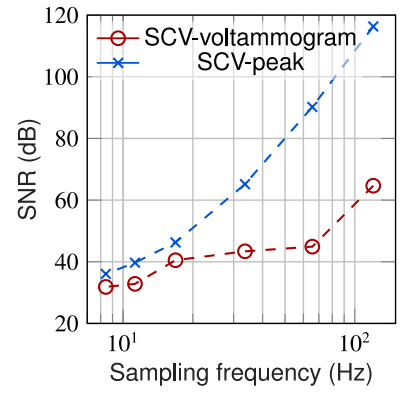

(b)
Fig. 2. Analysis of SCV output varying sampling rate. (a) Peak error percentage (w.r.t maximum). (b) SNR ratio (w.r.t. maximum) of full voltammogram (SCV-voltammogram) and detected peaks (SCV-peak).

\section{B. Reduction of Sampling Rate}

For the final analysis, two sensing methods are taken into account, SCV and DPV. The data collected using the seven-points calibration are analyzed progressively halving the sampling frequency from $33 \mathrm{~Hz}$ down to $0.1 \mathrm{~Hz}$. The selected range of frequency is derived from the literature and instrument (Autolab) indications [19]. The output current is re-sampled with an increasingly higher sampling interval $\left(\mathrm{t}_{\text {sample }}\right)$. Otherwise, the input driving voltage ramp is unvaried, to avoid any effect related to the increase of the voltage step $\left(\mathrm{V}_{\text {step }}\right)$. The down-sampling procedure visibly reduces the quantity of information in the voltammogram. Fig. 3 presents some points of the analysis for demonstration purpose. Fig. 4 reports graphically the results of this analysis from $33 \mathrm{~Hz}$ down to $0.6 \mathrm{~Hz}$. The results below this frequency are not valid since it is not possible to retrieve anymore useful information from the voltammogram. The parameters under investigation are the sensitivity (4a), the LOD (4b), the current peak position (4c), and the linearity of the calibration curves (4d).

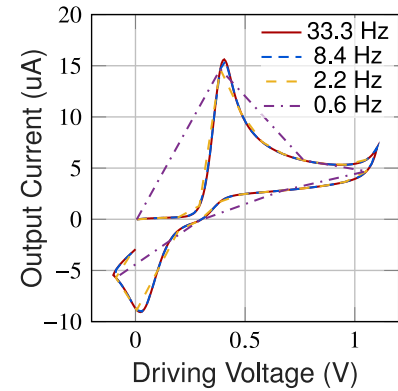

(a) (b)

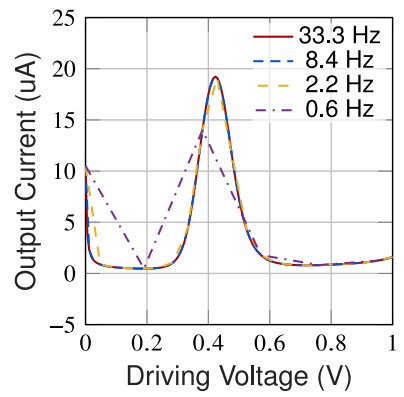

Fig. 3. Resulting voltammograms in SCV (a) and DPV (b), varying the sample-rate.

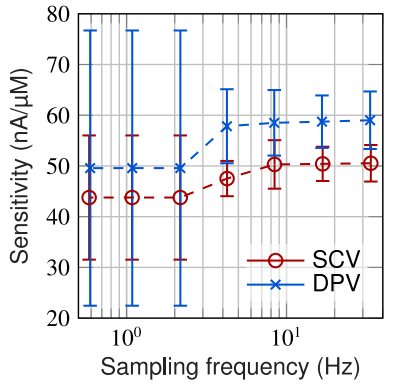

(a)

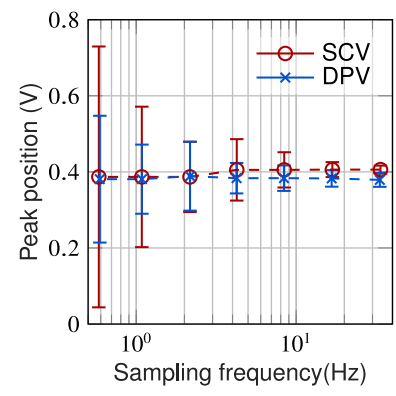

(c)

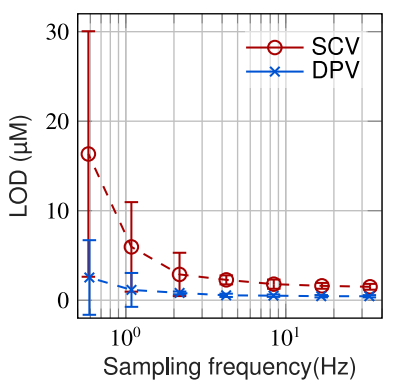

(b)

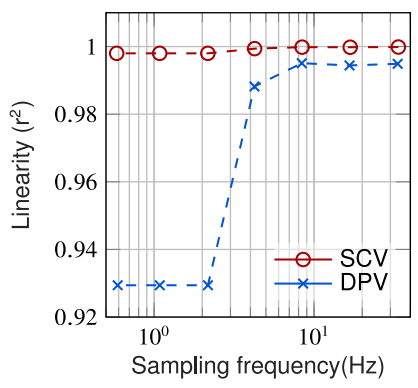

(d)
Fig. 4. Effect of down-sampling w.r.t. sampling frequency in SCV and DPV: (a) sensitivity, (b) LOD, (c) voltage peak position, (d) calibration curve linearity. 
TABLE 1. Comparison between empirical-defined sampling rate approach and the proposed optimal sampling rate approach.

\begin{tabular}{lcccc}
\hline \hline & \multicolumn{2}{c}{ Empirical approach } & \multicolumn{2}{c}{ Proposed approach } \\
& SCV & DPV & SCV & DPV \\
\hline Number of sample & 404 & 338 & 51 & 22 \\
Sampling frequency $(\mathrm{Hz})$ & 33.3 & 33.3 & 4.2 & 2.2 \\
Sensitivity $(\mathrm{nA} / \mu \mathrm{M})$ & $50.5 \pm 3.6$ & $59.0 \pm 5.7$ & $47.5 \pm 3.5$ & $57.8 \pm 7.3$ \\
LOD $(\mu \mathrm{M})$ & $1.50 \pm 0.11$ & $0.45 \pm 0.04$ & $2.27 \pm 0.16$ & $1.15 \pm 0.60$ \\
Peak position $(\mathrm{mV})$ & $406 \pm 11$ & $379 \pm 18$ & $405 \pm 80$ & $383 \pm 40$ \\
Linearity * & 0.999 & 0.995 & 0.999 & 0.988 \\
\hline \hline
\end{tabular}

${ }^{*}$ Coefficient of determination, $r^{2}$

The measurement errors are evaluated from the experimental data considering three times the standard deviation on each computed parameters. In all the graphs the measurement error increases visibly with the reduction of the sampling rate. The DPV method compensates the sensing-errors applying a differential detection and consequently enhances the LOD. The peak position remains stable varying its resolution, proving the selectivity. The linearity shows a drop in the DPV method below $2.2 \mathrm{~Hz}$, defining a clear limit in the proposed experimental set-up.

Considering the data reported in Fig. 4, is possible to find the right trade-off which minimizes the sampling rate, without affecting the performance of drug-sensing. Since the minimum therapeutic range of paracetamol of $50 \mu \mathrm{M}$, we define that in the optimal approach the LOD cannot exceed one order of magnitude this value (i. e. $5 \mu \mathrm{M}$ is the minimum LOD requirement). Moreover, we consider the drop in the linearity of DPV and we force the maximum S uncertainty to be lower than $10 \%$. We derive from the literature that usually the experiments are conducted with an empirical a priori defined sampling rate of more than some hundred samples per voltammogram [6]-[9], [19]. Table 1 shows a comparison between the empirical approach and the here presented approach. Thanks to the here presented optimization methodology, the LOD increases but remains in all cases under the imposed maximum. Sensitivity scales down to $94 \%$ of the standard with SCV method, and to $97 \%$ with DPV. Meanwhile, the sampling frequency can be reduced by eight times in the SCV and sixteen times in the DPV. If we consider the DPV method and our approach, this reflects on an astonishing reduction down to $6.6 \%$ of the sampling frequency, with a LOD still suitable for the application, loosing just $2 \%$ in sensitivity.

\section{CONCLUSION}

In the presented work, the results demonstrated how we can balance the performance to appropriate sampling rate and power consumption in voltammetry-based electrochemical sensing. Lowering the sampling rate the power consumption of the device diminishes, helping the realization of optimized electronic platforms able to provide non-invasive real-time point-of-care bio-sensing, targeting IoT and wearable applications. In particular, we established that is possible to develop a method to reduce the sampling frequency maintaining all the sensing performance in the range of the system requirements.

\section{ACKNOWLEDGMENTS}

This work is supported by Politecnico di Torino and Compagnia di San Paolo under the initiative "Joint research projects with top universities". The authors would like to thank Mr Riccardo Peloso for his helpful advice.

\section{REFERENCES}

[1] S. Nayak, N. R. Blumenfeld, T. Laksanasopin, and S. K. Sia, "Point-of-care diagnostics: Recent developments in a connected age," Analytical Chemistry, vol. 89, no. 1, pp. 102-123, Jan 2017.

[2] A. Tobola, F. J. Streit, C. Espig, O. Korpok, C. Sauter, N. Lang, B. Schmitz, C. Hofmann, M. Struck, C. Weigand, H. Leutheuser, B. M. Eskofier, and G. Fischer, "Sampling rate impact on energy consumption of biomedical signal processing systems," 2015 IEEE 12th International Conference on Wearable and Implantable Body Sensor Networks (BSN), pp. 1-6, June 2015.

[3] I. N. Hanitra, L. Lobello, F. Stradolini, A. Tuoheti, F. Criscuolo, T. Kilic, D. Demarchi, S. Carrara, and G. De Micheli, "A flexible front-end for wearable electrochemical sensing," 2018 IEEE International Symposium on Medical Measurements and Applications (MeMeA), pp. 1-6, June 2018.

[4] X. Xuan, H. S. Yoon, and J. Y. Park, "A wearable electrochemical glucose sensor based on simple and low-cost fabrication supported micro-patterned reduced graphene oxide nanocomposite electrode on flexible substrate," Biosensors and Bioelectronics, vol. 109, pp. 75-82, 2018

[5] M. W. Shinwari, D. Zhitomirsky, I. A. Deen, P. R. Selvaganapathy, M. J. Deen, and D. Landheer, "Microfabricated reference electrodes and their biosensing applications," Sensors, vol. 10, no. 3, pp. 1679-1715, 2010.

[6] F. Scholz, "Voltammetric techniques of analysis: the essentials," ChemTexts, vol. 1, no. 4, pp. 17, Sept 2015.

[7] F. Stradolini, A. Tuoheti, P. Motto Ros, D. Demarchi, and S. Carrara, "Raspberry Pi based system for portable and simultaneous monitoring of anesthetics and therapeutic compounds," 2017 New Generation of CAS (NGCAS), pp. 101-104, Sept 2017.

[8] A. Ainla, M. P. S. Mousavi, M.-N. Tsaloglou, J. Redston, J. G. Bell, M. T. FernándezAbedul, and G. M. Whitesides, "Open-source potentiostat for wireless electrochemical detection with smartphones," Analytical Chemistry, vol. 90, no. 10, pp. 6240-6246, 2018.

[9] I. Siegl, C. Kollegger, C. Rabl, C. Steffan, and W. Pribyl, "NFC powered cyclic voltammetry with dynamic output voltage range exploitation," 2018 12th International Conference on Sensing Technology (ICST), pp. 407-412, Dec 2018.

[10] S. Carrara, Bio/CMOS Interfaces and Co-Design, 1st ed. New York: SpringerVerlag, 2013.

[11] F. Stradolini, T. Elboshra, A. Biscontini, G. De Micheli, and S. Carrara, "Simultaneous monitoring of anesthetics and therapeutic compounds with a portable multichannel potentiostat," 2016 IEEE International Symposium on Circuits and Systems (ISCAS), pp. 834-837, May 2016.

[12] A. N. Amos, J. G. Roberts, L. Qi, L. A. Sombers, and G. S. McCarty, "Reducing the sampling rate of biochemical measurements using fast-scan cyclic voltammetry for in vivo applications," IEEE Sensors Journal, vol. 14, no. 9, pp. 2975-2980, Sept 2014.

[13] C. A. Lee, L. Qi, A. Amos, K. Blanton, G. S. McCarty, and L. A. Sombers, "Reducing data density in fast-scan cyclic voltammetry measurements of dopamine dynamics," Journal of The Electrochemical Society, vol. 165, no. 12, pp. 3042-3050, 2018.

[14] B. M. Kile, P. L. Walsh, Z. A. McElligott, E. S. Bucher, T. S. Guillot, A. Salahpour, M. G. Caron, and R. M. Wightman, "Optimizing the temporal resolution of fastscan cyclic voltammetry," ACS Chemical Neuroscience, vol. 3, no. 4, pp. 285-292, 2012.

[15] F. Prescott Laurie, "Paracetamol: Past, present, and future," American Journal of Therapeutics, vol. 7, no. 4, pp. 143-147, 2000.

[16] K. Aoki, J. Osteryoung, and R. A. Osteryoung, "Differential normal pulse voltammetry-theory," Journal of Electroanalytical Chemistry and Interfacial Electrochemistry, vol. 110, no. 1, pp. 1-18, 1980.

[17] Metrohm DropSens, Screen-Printed Carbon Electrodes Refs. 110, C110, C11L, 2019. Accessed: April 2019. [Online]. Available: www.dropsens.com/en/pdfs productos/new_brochures/110-c110-c111.pdf

[18] A. Bard, Electrochemical methods: fundamentals and applications. New York: Wiley, 2001

[19] Metrohm Autolab B.V., NOVA User manual, 2013. Accessed: April 2019. [Online] Available: metrohm-autolab.com/download/NovaSoftware/User_manual_1.10.pdf

[20] P. He, "Conversion of staircase voltammetry to linear sweep voltammetry by analog filtering," Analytical Chemistry, vol. 67, no. 5, pp. 986-992, 1995. 